JIRSS (2020)

Vol. 19, No. 01, pp 209-228

DOI:10.29252/jirss.19.1.209

\title{
The Weighted Exponentiated Family of Distributions: Properties, Applications and Characterizations
}

\author{
Zubair Ahmad ${ }^{1}$, Gholamhossien Hamedani ${ }^{2}$, and Mohammed Elgarhy ${ }^{3}$ \\ ${ }^{1}$ Department of Statistics, Quaid-i-Azam University 45320, Islamabad 44000, Pakistan. \\ ${ }^{2}$ Department of Mathematical and Statistical Sciences, Marquette University, Milwaukee, \\ WI 53201-1881, USA. \\ ${ }^{3}$ Valley High Institute for Management Finance and Information Systems, Obour, \\ Qaliubia, Egypt.
}

Received: 12/10/2018, Revision received: 16/07/2019, Published online: 26/10/2019

\begin{abstract}
In this paper a new method of introducing an additional parameter to a continuous distribution is proposed, which leads to a new class of distributions, called the weighted exponentiated family. A special sub-model is discussed. General expressions for some of the mathematical properties of this class such as the moments, quantile function, generating function and order statistics are derived; and certain characterizations are also discussed. To estimate the model parameters, the method of maximum likelihood is applied. A simulation study is carried out to assess the finite sample behavior of the maximum likelihood estimators. Finally, the usefulness of the proposed method via two applications to real data sets is illustrated.
\end{abstract}

Keywords. Exponentiated Family, Weighted Family, Exponential Distribution, Moments, Order Statistics, Characterizations, Maximum Likelihood Estimation.

Zubair Ahmad (z.ferry21@gmail.com)

Corresponding Author: Gholamhossien Hamedani (gholamhoss.hamedani@marquette.edu)

Mohammed Elgarhy (m_elgarhy85@yahoo.com) 
MSC: 62E15; 62G30.

\section{Introduction}

Generally, statistical distributions such as exponential, Weibull, Rayleigh, Gamma and Pareto distributions are frequently used in modeling data. However, in some cases, these classical distributions are not flexible enough to accommodate different phenomena of nature. For example, when the researchers record the observations according to certain stochastic model, the recorded observations will not have the original distribution unless each observation is given an equal chance of being recorded. In such a case, the traditional distributions do not provide adequate results and there is a clear need of the weighted versions of these distributions. Also, the use of weighed distributions may be the initial choice of the researchers when the samples are selected with probability proportional to some measure of the unit size. For better results, the specification of the right model for the underlying data is essential.

The concept of the weighted distributions goes back to "the study of the effects of methods of ascertainment upon the estimation of frequencies" of Fisher (1934). In this paper, Fisher posed the problem of the specification of the right model for the underlying data. Since then, a number of articles have been published on this subject. For further detail, we refer the interested readers to Patil and Ord (1975), Patel and Rao [(1977) and (1978)], Rao [(1977) and (1985)], Gupta and Kirmani (1990), Patil (2002), Shahbaz et al. (2010), Ghitany et al. (2011), Shakhatreh (2012), Mahdy (2013), Mahdavi (2015) and Kilany (2016).

Recently, Gupta and Kundu (2009) introduced a new class of weighted exponential (WE) distributions. They considered a special model of the subject class called WE distribution defined by the cumulative distribution function (cdf) given below

$$
F(x ; \alpha, \gamma)=\frac{\alpha+1}{\alpha}\left\{1-e^{-\gamma x}-\frac{1}{1+\alpha} e^{-\gamma(1+\alpha) x}\right\}, \quad x \geq 0, \alpha, \gamma>0 .
$$

The probability density function (pdf) corresponding to (1.1) is

$$
f(x ; \alpha, \gamma)=\frac{\alpha+1}{\alpha} \gamma e^{-\gamma x}\left\{1-e^{-\alpha \gamma x}\right\}, \quad x>0 .
$$

In this article, we propose a new weighted class of distributions. The key goal of this research is to introduce an extra parameter to a family of lifetime distribution 
functions to bring more flexibility to the given family. We call this new method as a new weighted family of continuous distributions, called the WEx family. Some of it's statistical properties will be studied. Furthermore, the key motivations for using WEx family in the practice are the followings:

1. A very simple and convenient method of adding additional parameters to modify the existing distributions.

2. To improve the characteristics and flexibility of the existing distributions.

3. To introduce the extended version of the baseline distribution whose cdf, sf and hrf, have closed form.

4. To provide better fits than the competing modified models having higher number of parameters than the proposed model.

The rest of this article is unfolded as follows: The propose family and its characterizations are presented in Section 2. Section 3 offers a special sub-model of the new family. Some mathematical properties are derived in Section 4. The maximum likelihood estimates of the unknown parameters and simulation study are presented in Section

5. Two real life applications are discussed in section 6. Finally, Section 7 concludes the article.

\section{The Proposed Family and its Characterizations}

In this section, we introduce the WEx family. Furthermore, we provide certain characterizations of the WEx distribution.

\subsection{The Weighted Exponentiated Family}

Let $X$ be a continuous random variable with cdf given by

$$
F(x ; \alpha, \xi)=G(x ; \xi)^{\alpha} e^{1-G(x ; \xi)}, \quad x \in \mathbb{R},
$$

where, $\alpha \geq 1$ is a parameter and $G(x ; \xi)$ is the baseline cdf depending on the vector parameter $\xi$. Here, in (2.1), the exponentiated cdf is weighted by the quantity $e^{1-G(x ; \xi)}$. 
The pdf, survival function (sf), hazard rate function (hrf) and cumulative hazard function (chf) corresponding to (2.1), are given, respectively, by

$$
\begin{array}{ll}
f(x ; \alpha, \xi)=g(x ; \xi) G(x ; \xi)^{\alpha-1} e^{1-G(x ; \xi)}[\alpha-G(x ; \xi)], & x \in \mathbb{R}, \\
S(x ; \alpha, \xi)=1-G(x ; \xi)^{\alpha} e^{1-G(x ; \xi)}, & x \in \mathbb{R}, \\
h(x ; \alpha, \xi)=\frac{g(x ; \xi) G(x ; \xi)^{\alpha-1}[\alpha-G(x ; \xi)]}{G(x ; \xi)^{\alpha}}, & x \in \mathbb{R}, \\
H(x ; \alpha, \xi)=-\log \left\{1-G(x ; \xi)^{\alpha} e^{1-G(x ; \xi)}\right\}, & x \in \mathbb{R} .
\end{array}
$$

Henceforth, a random variable $X$ with the pdf given by (2.2) will be denoted by $X \sim W E x(\alpha, \xi)$. For the sake of simplicity, we can omit the dependence on the vector parameter $\xi$, and simply write $G(x ; \xi)=G(x)$.

\subsection{Characterizations}

This sub-section deals with the characterizations of the WEx distribution in different directions: (i) based on the ratio of two truncated moments; (ii) in terms of the reverse hazard function; and (iii) based on the conditional expectation of the certain function of the random variable. Note that for the characterization (i) the cdf does not need a closed form closed form and depends on the solution of a first order differential equation, which provides a bridge between probability and differential equation. We would also like to mention that due to the nature of the cdf of (2.1), our characterizations may be the only possible ones. We also like to mention that characterization (i) is stable in the sense of weak convergence (Glänzel,1990). We present our characterizations (i)-(iii) in three sub-subsections.

\subsubsection{Characterizations Based on Two Truncated Moments}

In this sub-subsection, we present the characterizations of WEx distribution based on the ratio of two truncated moments. Our first characterization employs a theorem due to Glänzel (1987); see Theorem 1 of Appendix A. The result, however, holds also when the interval $H$ is not closed, since the condition of the Theorem is on the interior of $H$.

Proposition 2.2.1. Let $X: \Omega \rightarrow \mathbb{R}$ be a continuous random variable and let $q_{1}(x)=$ $G(x)^{1-\alpha}(\alpha-G(x))^{-1}$ and $q_{2}(x)=q_{1}(x) e^{1-G(x)}$ for $x \in \mathbb{R}$. The random variable $X$ has pdf 
(2.2) if and only if the function $\eta(x)$ defined in Theorem 1 is of the form

$$
\eta(x)=\frac{1}{2}\left(e^{1-G(x)}-1\right), \quad x \in \mathbb{R} .
$$

Proof. Suppose the random variable $X$ has pdf (2.2), then

$$
(1-F(x)) E\left(q_{1}(X) \mid X \geq x\right)=e^{1-G(x)}-1, \quad x \in \mathbb{R},
$$

and

$$
(1-F(x)) E\left(q_{2}(X) \mid X \geq x\right)=\frac{1}{2}\left(e^{2(1-G(x))}-1\right), \quad x \in \mathbb{R} .
$$

Further,

$$
\eta(x) q_{1}(x)-q_{2}(x)=-\frac{q_{1}(x)}{2}\left(e^{1-G(x)}+1\right)<0, \quad x \in \mathbb{R} .
$$

Conversely, if $\eta(x)$ is of the above form, then

$$
s^{\prime^{\prime}}(x)=\frac{\eta^{\prime}(x) q_{1}(x)}{\eta(x) q_{1}(x)-q_{2}(x)}=\frac{g(x) e^{1-G(x)}}{e^{1-G(x)}+1}, \quad x \in \mathbb{R},
$$

and consequently

$$
s(x)=-\log \left\{e^{1-G(x)}+1\right\}, \quad x \in \mathbb{R} .
$$

Now, according to Theorem $1, X$ has density (2.2).

Corollary 2.2.1. Let $X: \Omega \rightarrow \mathbb{R}$ be a continuous random variable and let $q_{1}(x)$ be as in Proposition 2.2.1. The random variable $X$ has pdf (2.2) if and only if there exist functions $q_{2}(x)$ and $\eta(x)$ defined in Theorem 1 satisfying the following differential equation

$$
\frac{\eta^{\prime}(x) q_{1}(x)}{\eta(x) q_{1}(x)-q_{2}(x)}=\frac{g(x) e^{1-G(x)}}{e^{1-G(x)}+1}, \quad x \in \mathbb{R} .
$$

Corollary 2.2.2. The general solution of the differential equation in Corollary 2.2.1 is

$$
\eta(x)=\left\{e^{1-G(x)}+1\right\}^{-1}\left[-\int g(x) e^{1-G(x)}\left(q_{1}(x)\right)^{-1} q_{2}(x) d x+D\right],
$$

where $D$ is a constant. We like to point out that one set of functions satisfying the above differential equation is given in Proposition 2.2.1 with $D=1 / 2$. Clearly, there are other triplets $\left(q_{1}(x), q_{2}(x), \eta(x)\right)$ which satisfy conditions of Theorem 1 . 


\subsubsection{Characterization in Terms of the Reverse Hazard Function}

The reverse hazard function, $r_{F}(x)$ of a twice differentiable distribution function, $F(x)$, is defined as

$$
r_{F}(x)=\frac{f(x)}{F(x)}, \quad x \in \text { support of } F(x) .
$$

In this sub-subsection we present a characterization of WEx distribution in terms of the reverse hazard function.

Proposition 2.2.2 Let $X: \Omega \rightarrow \mathbb{R}$ be a continuous random variable. The random variable $X$ has pdf (2.2) if and only if its reverse hazard function $r_{F}(x)$ satisfies the following differential equation

$$
r_{F}^{\prime}(x)+g(x) r_{F}(x)=G(x)^{-2} e^{1-G(x)}\left[g^{\prime}(x) G(x)\{\alpha-G(x)\}-\alpha g(x)^{2}\right], \quad x \in \mathbb{R} .
$$

Proof. If $X$ has density (2.2), then clearly the above differential equation holds. Now, if the differential equation holds, then

$$
\frac{d}{d x}\left\{g(x) e^{G(x)-1}\right\}=\frac{d}{d x}\left\{G(x)^{-1}(\alpha-G(x))\right\}, \quad x \in \mathbb{R},
$$

from which we obtain the reverse hazard function of (2.2).

\subsubsection{Characterization Based on the Conditional Expectation of Certain Functi- on of the Random Variable}

In this sub-subsection we employ a single function $\psi$ of $X$ and characterize the distribution of $X$ in terms of the truncated moment of $\psi(X)$. The following proposition has already appeared in Hamedani's previous work (2013), so we will just state it here which can be used to characterize the WEx distribution.

Proposition 2.2.3. Let $\mathrm{X}: \Omega \rightarrow(e, f)$ be a continuous random variable with cdf $F$. Let $\psi(x)$ be a differentiable function on $(e, f)$ with $\lim _{x \rightarrow f^{-}} \psi(x)=1$. Then for $\delta \neq 1$,

$$
E[\psi(X) \mid X \leq x]=\delta \psi(x), \quad x \in(e, f),
$$

implies

$$
\psi(x)=(F(x))^{\frac{1}{\delta}-1}, \quad x \in(e, f) .
$$

Remark 2.3.1. For $(e, f)=\mathbb{R}, \psi(x)=G(x) e^{\frac{(1-G(x))}{\alpha}}$ and $\delta=\frac{\alpha}{\alpha+1}$, Proposition 2.2 .3 provides a characterization of WEx distribution. 


\section{The Weighted Exponentiated Exponential Distrib- ution}

Consider the distribution and density functions of the exponential random variable given by $g(x)=\gamma e^{-\gamma x}, \quad x, \gamma>0$, and $G(x)=1-e^{-\gamma x}$. Then, the cdf of the WEx-E distribution is given by

$$
F(x)=\left(1-e^{-\gamma x}\right)^{\alpha} e^{e^{-\gamma x}}, \quad x \geq 0, \gamma>0, \alpha \geq 1 .
$$

The pdf, sf, hf and chf corresponding to (3.1), are

$$
\begin{array}{ll}
f(x)=\gamma e^{-\gamma x}\left(1-e^{-\gamma x}\right)^{\alpha-1} e^{e^{-\gamma x}}\left[\alpha-1+e^{-\gamma x}\right], & x>0, \\
S(x)=1-\left(1-e^{-\gamma x}\right)^{\alpha} e^{e^{-\gamma x}}, & x>0, \\
h(x)=\frac{\gamma e^{-\gamma x}\left(1-e^{-\gamma x}\right)^{\alpha-1} e^{e^{-\gamma x}}\left[\alpha-1+e^{-\gamma x}\right]}{1-\left(1-e^{-\gamma x}\right)^{\alpha} e^{e^{-\gamma x}},} & x>0, \\
H(x)=-\log \left[1-\left(1-e^{-\gamma x}\right)^{\alpha} e^{e^{-\gamma x}}\right], & x>0 .
\end{array}
$$

Plots of the WEx-E density and hazard functions for selected parameter values are sketched in Figures 1 and 2, respectively.
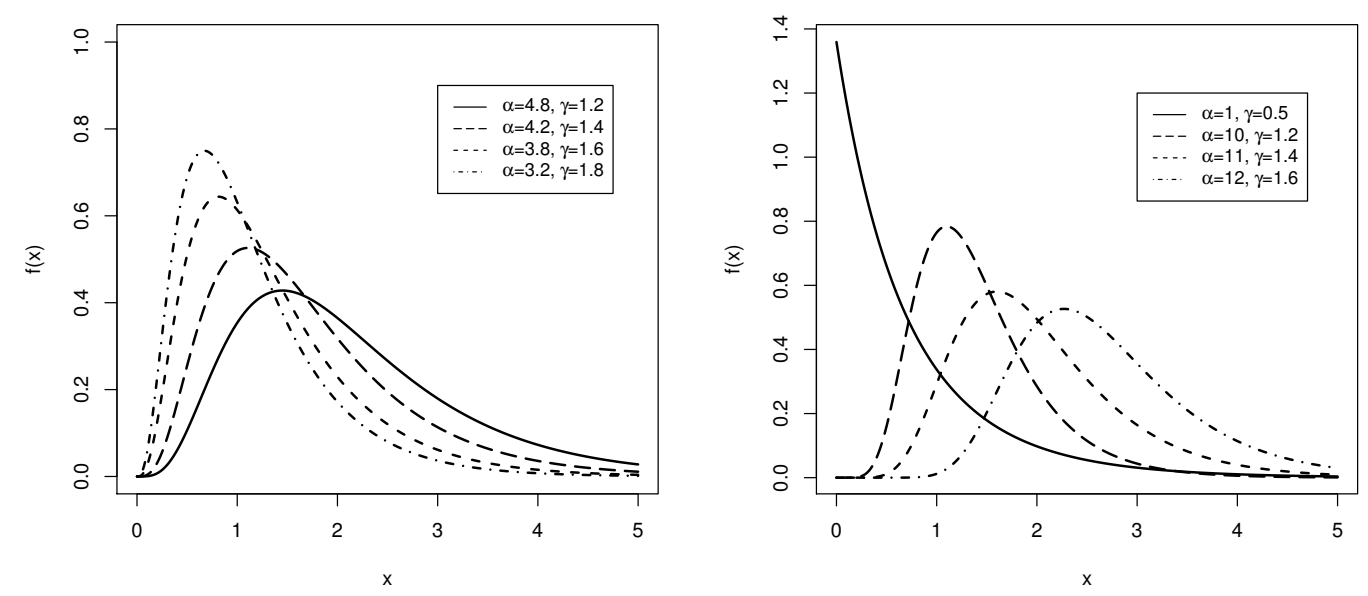

Figure 1: Plots of the density function of WEx-E distribution for selected parameter values. 

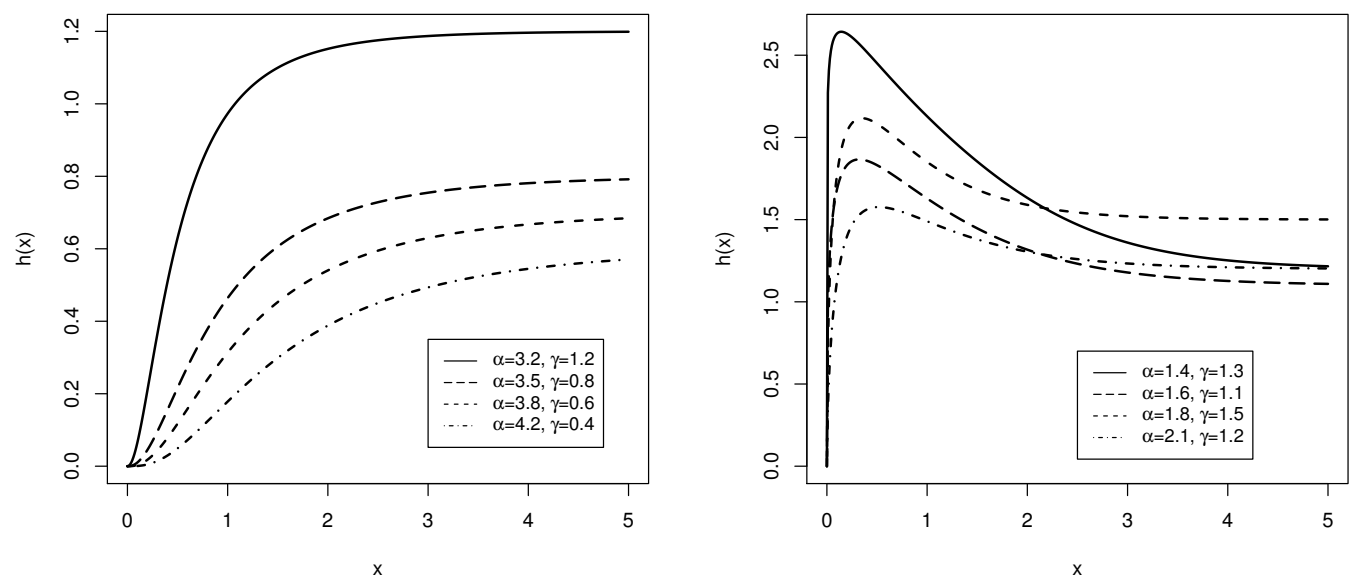

Figure 2: Plots of the hazard function of WEx-E distribution for some parameter values.

The WE distribution proposed by Gupta and Kundu (2007) is capable of modeling data with increasing hrf. But, it fails to model data with unimodal failure rate function (falls under the category of non-monotonic hrf) which is an important realization of the non-monotonic hrf. Whereas, the WEx-E is capable of modeling data with increasing hrf (see Figure 2) as well as with unimodal hrf which is very useful to determine the time period having maximum risk.

\section{Mathematical Properties}

In this section, we discuss some of the properties of the WEx family.

\subsection{Quantile Function}

The quantile function of the WEx random variable $X$ can be obtained by inverting $F\left(x_{q} ; \xi\right)=q$ in $(2.1)$. We obtain

$$
\log \left\{\frac{F(x ; \xi)^{\alpha}}{q}\right\}+1-F(x ; \xi)=0
$$


The expression (4.1) does not have a closed form for the solution in $x_{q}$, therefore, numerical methods are required to obtain the solution of (4.1).

\subsection{Moments}

Let $X$ follow the WEx family of distributions, then the $r^{\text {th }}$ moment of $X$ denoted by $\mu_{r}^{\prime}$ is

$$
\mu_{r}^{\prime}=\int_{-\infty}^{\infty} x^{r} f(x ; \alpha, \xi) d x
$$

Using (2.2) in (4.2), we have

$$
\mu_{r}^{\prime}=\sum_{i, j=0}^{\infty} \eta_{i, j} \int_{-\infty}^{\infty} x^{r}\left[\alpha \Lambda_{\alpha+j-1}-\Lambda_{\alpha+j}^{\prime}\right] d x,
$$

where, $\eta_{i, j}=\frac{1}{i !}\left(\begin{array}{c}i \\ j\end{array}\right), \Lambda_{\alpha+j-1}=g(x ; \xi) G(x ; \xi)^{\alpha+j-1}$ and $\Lambda_{\alpha+j}^{\prime}=g(x ; \xi) G(x ; \xi)^{\alpha+j}$. Finally, we get

$$
\mu_{r}^{\prime}=\sum_{i, j=0}^{\infty} \eta_{i, j}\left[\alpha \psi_{r, \alpha+j-1}-\psi_{r, \alpha+j}^{\prime}\right],
$$

where, $\psi_{r, \alpha+j-1}=\int_{-\infty}^{\infty} x^{r} g(x ; \xi) G(x ; \xi)^{\alpha+j-1} d x$ and $\psi_{r, \alpha+j}^{\prime}=\int_{-\infty}^{\infty} x^{r} g(x ; \xi) G(x ; \xi)^{\alpha+j} d x$.

Furthermore, the moment generating function of $X, M_{x}(t)$, is given by

$$
M_{x}(t)=\sum_{r, i, j=0}^{\infty} \kappa_{r, i, j}\left[\alpha \psi_{r, \alpha+j-1}-\psi_{r, \alpha+j}^{\prime}\right],
$$

where, $\kappa_{r, i, j}=\frac{t^{r}}{r ! i !}\left(\begin{array}{l}i \\ j\end{array}\right)$.

\subsection{Probability Weighted Moments}

The probability weighted moments (pwm) is another useful approach to describe the most important feature of the distribution. Here, we derive the pwm, denoted by $\tau_{r, m}$, of $X$ as follows

$$
\tau_{r, m}=\int_{-\infty}^{\infty} x^{r} f(x ; \alpha, \xi) F(x ; \alpha, \xi)^{m} d x
$$


Inserting (B-4) in (4.5), we have

$$
\tau_{r, m}=\sum_{i, j, k=0}^{\infty} \Upsilon_{m, i, j, k}\left[\alpha v_{r, \alpha(m+1) j+k-1}-v_{r, \alpha(m+1) j+k}^{\prime}\right],
$$

where, $v_{r, \alpha(m+1) j+k-1}=\int_{-\infty}^{\infty} x^{r} v_{\alpha(m+1) j+k-1} d x$ and $v_{r, \alpha(m+1) j+k}^{\prime}=\int_{-\infty}^{\infty} x^{r} v_{\alpha(m+1) j+k}^{\prime} d x$.

\subsection{Residual Life}

The residual life plays a vital role in practice, particularly in reliability theory. The remaining waiting time for an event to on the condition that we have already been waiting is what we call residual life. Let $X$ follow WEx family of distributions, then the residual life of $X$ is

$$
\begin{gathered}
\varepsilon(x)=\frac{S(x+t)}{S(x)}, \\
\varepsilon(x)=\frac{F(x+t)^{\alpha} e^{1-F(x+t)}}{F(x)^{\alpha} e^{1-F(x)} .} .
\end{gathered}
$$

\subsection{Reverse Residual Life}

The reverse residual life of a lifetime random variable is of interest in many areas of applied sciences such as survival analysis, actuarial studies and risk management. The reverse residual lifetime of $X$ denoted by $\bar{\varepsilon}(x)$ is

$$
\begin{gathered}
\bar{\varepsilon}(x)=\frac{S(x-t)}{S(x)}, \\
\bar{\varepsilon}(x)=\frac{F(x-t)^{\alpha} e^{1-F(x-t)}}{F(x)^{\alpha} e^{1-F(x)} .} .
\end{gathered}
$$

\subsection{Order Statistics}

The order statistics have wider applications in many applied areas of statistics. Suppose $x_{1}, x_{2}, \ldots, x_{n}$ are the observed values of a random sample $X_{1}, X_{2}, \ldots, X_{n}$ taken from the WEx family. The density function of the $r^{\text {th }}$ order statistic, say $X_{r: n}$, is

$$
f_{r: n}(x)=\frac{n !}{(l-1) !(n-l) !} \sum_{l=0}^{n-r}(-1)^{l}\left(\begin{array}{c}
n-r \\
l
\end{array}\right) f(x ; \alpha, \xi) F(x ; \alpha, \xi)^{l+r-1} .
$$


The pdf of the $r^{\text {th }}$ order statistic of WEx family is derived by using (B-4) in (4.7), and replacing $\mathrm{m}$ with $l+r-1$

$$
g_{r: n}(x)=\frac{n !}{(l-1) !(n-l) !} \sum_{i, j, k, l=0}^{n-r}(-1)^{l}\left(\begin{array}{c}
n-r \\
l
\end{array}\right) \Upsilon_{l+r, i, j, k-1}\left[\alpha v_{\alpha(l+r) j+k-1}-v_{\alpha(l+r) j+k}^{\prime}\right] .
$$

Based on the expansion (4.8), we can derive some of the mathematical properties (such as incomplete and ordinary moments generating function, etc.) for the WEx order statistics.

\section{Maximum Likelihood Estimation and Simulation}

In this section, we discuss the estimation of the unknown parameters of the WEx family via the method of maximum likelihood. Also, we provide a simulation to assess the behavior of the maximum likelihood estimators.

\subsection{Maximum Likelihood Estimation}

Let $x_{1}, x_{1}, \ldots, x_{n}$ be the observed values of a random sample from pdf (2.2) with parameters $\alpha$ and $\xi$. The log-likelihood function is

$$
\begin{aligned}
\log L(x ; \alpha, \xi) & =\sum_{i=1}^{n} \log \left[g\left(x_{i} ; \xi\right)\right]+(\alpha-1) \sum_{i=1}^{n} \log \left[G\left(x_{i} ; \xi\right)\right] \\
& +\sum_{i=1}^{n}\left[1-G\left(x_{i} ; \xi\right)\right]+\sum_{i=1}^{n} \log \left[\alpha-G\left(x_{i} ; \xi\right)\right]
\end{aligned}
$$

The partial derivatives of the function $\log L(x ; \alpha, \xi)$ are

$$
\begin{aligned}
\frac{\partial}{\partial \alpha} \log L(x ; \alpha, \xi) & =\sum_{i=1}^{n} \log \left[G\left(x_{i} ; \xi\right)\right]+\sum_{i=1}^{n} \frac{1}{\alpha-G\left(x_{i} ; \xi\right)} \\
\frac{\partial}{\partial \xi} \log L(x ; \alpha, \xi) & =\sum_{i=1}^{n} \frac{\partial g\left(x_{i} ; \xi\right) / \partial \xi}{g\left(x_{i} ; \xi\right)}+(\alpha-1) \sum_{i=1}^{n} \frac{\partial G\left(x_{i} ; \xi\right) / \partial \xi}{G\left(x_{i} ; \xi\right)} \\
& -\sum_{i=1}^{n} \partial G\left(x_{i} ; \xi\right) / \partial \xi-\sum_{i=1}^{n} \frac{\partial G\left(x_{i} ; \xi\right) / \partial \xi}{\alpha-G\left(x_{i} ; \xi\right)}
\end{aligned}
$$

The maximum likelihood estimates of $\alpha$ and $\xi$ are numerical solutions of (5.2) and (5.3) simultaneously. 


\subsection{Simulation Study}

In this sub-section, we take up the simulation study for WEx-E distribution with parameters $\alpha$ and $\gamma$. The process is described below:

1. Random samples of sizes $n=100,150,200$ and 300 are generated from WEx-E distribution and parameters have been estimated via the maximum likelihood method.

2. 1000 repetitions are made to calculate the bias, mean square error (MSE) and root mean square error (RMSE) of these estimators.

3. Formulas used for calculating Bias and RMSE are given by

$$
\operatorname{Bias}(\hat{\alpha})=\frac{1}{1000} \sum_{i=1}^{1000}\left(\hat{\alpha}_{i} \alpha\right) \text { and } \operatorname{RMSE}(\hat{\alpha})=\sqrt{\frac{1}{1000} \sum_{i=1}^{1000}\left(\hat{\alpha}_{i}-\alpha\right)^{2}} \text {, respectively. }
$$

4. Step 3 is also repeated for the other parameter $\gamma$.

The results are provided in Tables 1-3.

Table 1: The simulation results of the WEX-E model using maximum likelihood method.

\begin{tabular}{cccccccccc}
\hline & & \multicolumn{3}{c}{ Set $1: \alpha=1.5, \gamma=0.3$} & & \multicolumn{3}{c}{ Set 2: $\alpha=1.2, \gamma=0.3$} \\
\cline { 3 - 5 } \cline { 8 - 10 }$n$ & Par & MLE & Bais & RMSE & & MLE & Bais & RMSE \\
\hline \multirow{2}{*}{100} & $\alpha$ & 4.732 & 3.232 & 3.344 & & 3.663 & 2.463 & 2.548 \\
& $\gamma$ & 1.407 & 1.107 & 1.115 & & 1.895 & 1.595 & 1.619 \\
\hline 150 & $\alpha$ & 3.337 & 1.837 & 1.879 & & 2.777 & 1.577 & 1.616 \\
& $\gamma$ & 0.971 & 0.671 & 0.678 & & 1.217 & 0.917 & 0.925 \\
\hline 200 & $\alpha$ & 2.600 & 1.100 & 1.120 & & 2.203 & 1.003 & 1.005 \\
& $\gamma$ & 0.693 & 0.393 & 0.398 & & 0.937 & 0.637 & 0.645 \\
\hline 300 & $\alpha$ & 1.338 & -0.162 & 0.377 & & 1.222 & 0.022 & 0.256 \\
& $\gamma$ & 0.251 & -0.049 & 0.109 & & 0.308 & 0.008 & 0.092 \\
\hline
\end{tabular}

Table 2: The simulation results of the WEx-E model using maximum likelihood method.

\begin{tabular}{ccccccccc}
\hline & & \multicolumn{3}{c}{ Set 3: $\alpha=1, \gamma=0.3$} & & \multicolumn{3}{c}{ Set 4: $\alpha=1.5, \gamma=0.1$} \\
\cline { 3 - 5 } \cline { 7 - 9 }$n$ & Par & MLE & Bais & RMSE & & MLE & Bais & RMSE \\
\hline \multirow{2}{*}{100} & $\alpha$ & 3.030 & 2.030 & 2.051 & & 5.082 & 3.582 & 3.636 \\
& $\gamma$ & 2.606 & 2.306 & 2.310 & & 0.468 & 0.368 & 0.372 \\
\hline \multirow{2}{*}{150} & $\alpha$ & 2.474 & 1.474 & 1.486 & & 3.155 & 1.655 & 1.669 \\
& $\gamma$ & 1.676 & 1.376 & 1.384 & & 0.300 & 0.200 & 0.201 \\
\hline \multirow{2}{*}{200} & $\alpha$ & 1.939 & 0.939 & 0.949 & & 2.538 & 1.038 & 1.050 \\
& $\gamma$ & 1.188 & 0.888 & 0.894 & & 0.231 & 0.131 & 0.133 \\
\hline 300 & $\alpha$ & 1.041 & 0.041 & 0.078 & & 1.333 & -0.167 & 0.415 \\
& $\gamma$ & 0.329 & 0.029 & 0.059 & & 0.082 & -0.018 & 0.040 \\
\hline
\end{tabular}


Table 3: The simulation results of the WEx-E model using maximum likelihood method.

\begin{tabular}{cccccccccc}
\hline & & \multicolumn{3}{c}{ Set 5: $\alpha=2.1, \gamma=0.7$} & & \multicolumn{3}{c}{ Set $6: \alpha=1.8, \gamma=0.7$} \\
\cline { 3 - 5 } \cline { 8 - 10 }$n$ & Par & MLE & Bais & RMSE & & MLE & Bais & RMSE \\
\hline \multirow{2}{*}{100} & $\alpha$ & 6.771 & 4.671 & 4.795 & & 5.543 & 3.743 & 4.017 \\
& $\gamma$ & 2.430 & 1.730 & 1.744 & & 2.825 & 2.125 & 2.144 \\
\hline 150 & $\alpha$ & 5.223 & 3.123 & 3.188 & & 4.024 & 2.224 & 2.267 \\
& $\gamma$ & 1.846 & 1.146 & 1.151 & & 2.026 & 1.326 & 1.339 \\
\hline \multirow{2}{*}{200} & $\alpha$ & 3.517 & 1.417 & 1.443 & & 3.037 & 1.237 & 1.308 \\
& $\gamma$ & 1.250 & 0.550 & 0.559 & & 1.369 & 0.669 & 0.690 \\
\hline \multirow{2}{*}{300} & $\alpha$ & 1.141 & -0.959 & 1.156 & & 1.090 & -0.710 & 0.887 \\
& $\gamma$ & 0.291 & -0.409 & 0.482 & & 0.326 & -0.374 & 0.454 \\
\hline
\end{tabular}

\section{Applications}

In this section, we illustrate the proposed family by considering the WEx-E distribution and compare the results with the exponentiated Weibull (EW), Marshall-Olkin Weibull (MOW), alpha power transformed Weibull (APTW) and Kumaraswamy Weibull (KuW) distributions by means of analyzing two real applications. The analytical measures of the goodness of fit including the Akaike information criterion (AIC), consistent Akaike information criterion (CAIC), Bayesian information criterion (BIC), HannanQuinn information criterion (HQIC), Kolmogorov-Smirnov (KS), Cramer-von Mises $(\mathrm{CM})$ and Anderson-Darling (AD) statistics are considered to compare the proposed method with the fitted models. The statistics $\mathrm{CM}$ and AD are described in details in Chen and Balakrishnan (1995). In general, a model with smaller values of these analytical measure indicate better fit to the data. All the required computations have been done in the R-language using SANN algorithm.

Data 1: The data set represents survival times of guinea pigs injected with different amount of tubercle bacilli studied by Bjerkedal (1960). Guinea pigs are subject to high susceptibility of human tuberculosis, which is one of the causes for choosing this species. We take into account only the study in which animals in a single cage are under the same regimen. The data represents the survival times of Guinea pigs in days which are listed as: 10, 33, 44, 56, 59, 72, 74, 77, 92, 93, 96, 100, 100, 102, 105, 107, 107, $108,108,108,109,112,113,115,116,120,121,122,122,124,130,134,136,139,144$, $146,153,159,160,163,163,168,171,172,176,183,195,196,197,202,213,215,216,222$, $230,231,240,245,251,253,254,255,278,293,327,342,347,361,402,432,458,555$. Table 
4 lists the MLEs and the considered statistics are listed in table 5. Corresponding to data 1, the estimated pdf and cdf are plotted in Figure 3.

Table 4: Maximum likelihood estimates of the fitted distributions using data 1.

\begin{tabular}{lllllll}
\hline Dist. & $\hat{\alpha}$ & $\hat{\gamma}$ & $\hat{\theta}$ & $\hat{\lambda}$ & $\hat{a}$ & $\hat{b}$ \\
\hline WEx $-E$ & 4.140 & 0.010 & & & & \\
MOW & & 0.003 & 1.212 & 4.043 & & \\
APTW & 15.315 & 0.0043 & 1.154 & & & \\
Ku-W & & 0.006 & 1.063 & & 2.885 & 1.104 \\
EW & 4.1107 & 0.015 & 0.948 & & & \\
\hline
\end{tabular}

Table 5: The statistics of the fitted models using data 1.

\begin{tabular}{llllllll}
\hline Dist. & KS & CM & AD & AIC & BIC & CIAC & HQIC \\
\hline WEx-E & 0.091 & 0.076 & 0.509 & 856.38 & 860.93 & 856.56 & 858.19 \\
MOW & 0.123 & 0.191 & 1.113 & 863.57 & 870.40 & 863.92 & 866.29 \\
APTW & 0.138 & 0.134 & 0.779 & 860.67 & 867.50 & 861.02 & 863.39 \\
Ku-W & 0.100 & 0.086 & 0.543 & 859.49 & 868.595 & 860.08 & 863.11 \\
EW & 0.093 & 0.078 & 0.517 & 857.88 & 864.71 & 858.24 & 860.60 \\
\hline
\end{tabular}

Data 2: The second data set taken from Lawless (2011), represents the length of lifetime of thirteen ball bearing recorded from a life testing experiment of deep groove ball bearings. The data are as follow: 17.88, 28.92, 33.00, 41.52, 42.12, 45.60, 48.80, 51.84, 51.96, 54.12, 55.56, 67.80, 68.64, 68.64, 68.88, 84.12, 93.12, 98.64, 105.12, 105.84, 127.92, 128.04, 173.40. Table 6 provides the MLEs and the considered statistics are given in table 7. Corresponding to data 2, the estimated pdf and cdf are plotted in Figure 4.

Table 6: Maximum likelihood estimates of the fitted distributions using data 2.

\begin{tabular}{lllllll}
\hline Dist. & $\hat{\alpha}$ & $\hat{\gamma}$ & $\hat{\theta}$ & $\hat{\lambda}$ & $\hat{a}$ & $\hat{b}$ \\
\hline WEx $-E$ & 5.853 & 0.031 & & & & \\
MOW & & 0.005 & 1.380 & 4.658 & & \\
APTW & 8.0731 & 0.003 & 1.395 & & & \\
Ku-W & & 0.028 & 1.084 & & 5.709 & 0.691 \\
EW & 5.431 & 0.0332 & 0.997 & & & \\
\hline
\end{tabular}



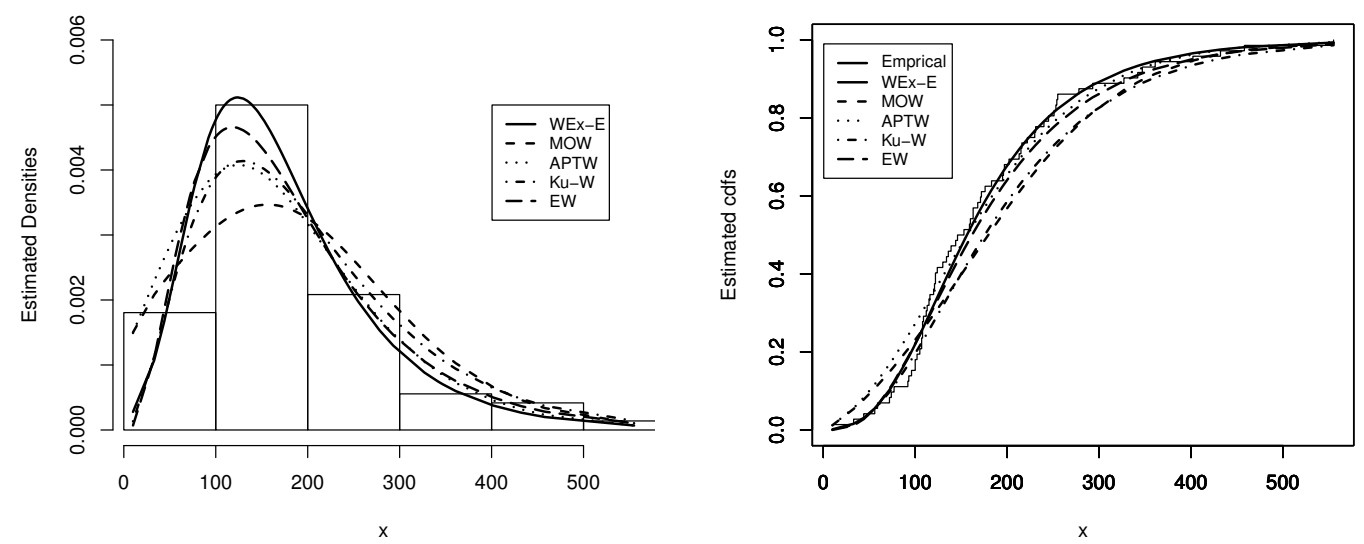

Figure 3: Estimated pdf and cdf of the WEx-E distribution for data 1.

Table 7: The analytical measures of the fitted distributions using data 2.

\begin{tabular}{llllllll}
\hline Dist. & KS & CM & AD & AIC & BIC & CIAC & HQIC \\
\hline WEx-E & 0.105 & 0.031 & 0.186 & 229.95 & 232.22 & 230.55 & 230.52 \\
MOW & 0.125 & 0.078 & 0.438 & 234.79 & 238.20 & 236.06 & 235.65 \\
APTW & 0.133 & 0.060 & 0.334 & 234.39 & 237.80 & 235.65 & 235.25 \\
Ku-W & 0.120 & 0.035 & 0.189 & 233.99 & 238.53 & 236.21 & 235.13 \\
EW & 0.109 & 0.032 & 0.188 & 231.95 & 235.36 & 233.22 & 232.81 \\
\hline
\end{tabular}

\section{Conclusions}

A new family of distributions, called the weighted exponentiated family is proposed and studied. General expressions for some of the properties of the new family including moments, moment generating function, quantile, residual life, reverse residual life and order statistics are derived. The advantage of using this family is that its cdf has a closed form solution anda facilitating data modeling with monotonic and nonmonotonic failure rates. The maximum likelihood method is used to estimate the model 
parameters. For the evaluation of the maximum likelihood estimators, a simulation study is provided. Certain characterizations of the proposed family are also provided. A special sub-model of this family, called the weighted exponentiated exponential distribution is considered and its real applications are analyzed. Empirically, it is proved that the special sub-model can provide a better fit in modeling data than the other competing distributions.
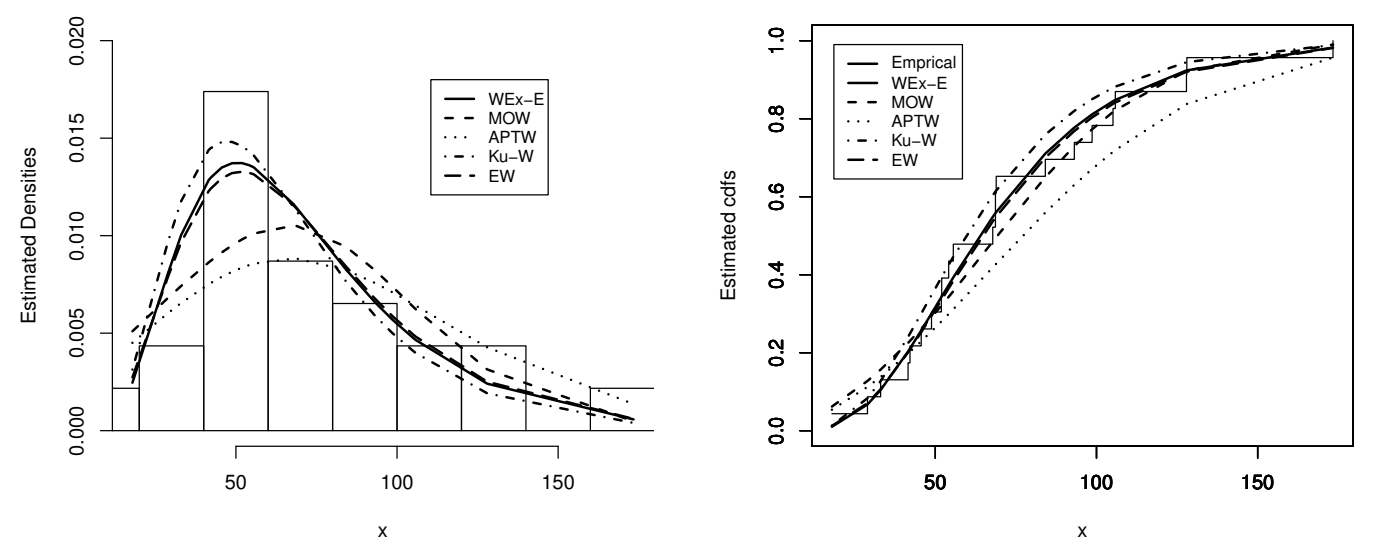

Figure 4: Estimated pdf and cdf of the WEx-E distribution for data 2.

\section{Acknowledgements}

The authors are grateful to the Editor-in-Chief, the Associate Editor and anonymous referees for many of their valuable comments and suggestions which has led to this improved version of the manuscript.

\section{References}

Bjerkedal, T. (1960), Acquisition of Resistance in Guinea Pies infected with Different Doses of Virulent Tubercle Bacilli. American Journal of Hygiene, 72, 130-48. 
Fisher, R. A. (1934), The effects of methods of ascertainment upon the estimation of frequellcies. Annals of Eugenics, 6, 13-25.

Ghitany, M. E., Al-qallaf, F., Al-Mutairi, D. K. and Husain, H. A. (2011), A twoparameter weighted Lindley distribution and its applications to survival data. Mathematics and computers in Simulation, 81, 1190-1201.

Glänzel, W. (1987), A characterization theorem based on truncated moments and its application to some distribution families, Mathematical Statistics and Probability Theory, B, 75-84.

Glänzel, W. (1990), Some consequences of a characterization theorem based on truncated moments, Statistics: A Journal of Theoretical and Applied Statistics, 21, 613618.

Gupta, R. and Kirmani, S. (1990), The role of weighted distribution in stochastic modeling. Communication in Statistics: Theory \& Methods, 19, 3147-3162.

Gupta, R. D. and Kundu, D. (2009), A new class of weighted exponential distributions. Statistics, 43, 621-634.

Hamedani, G. G. (2013), On certain generalized gamma convolution distributions II, Technical Report No. 484, MSCS, Marquette University.

Kilany, N. M. (2016), Weighted Lomax distribution. Springer Plus, 5, 1-18.

Lawless, J. F. (2011), Statistical models and methods for lifetime data. John Wiley E Sons, 362.

Mahdavi, A. (2015), Two weighted distributions generated by exponential distribution. Journal of Mathematical Extension, 9, 1-12.

Mahdy, M. (2013), A class of weighted Weibull distributions and its properties. Studies in Mathematical Sciences, 6, 35-45. 
Patil, G. P. (2002), Weighted distributions.Encyclopedia of Environmetics, 4, 2369-2377.

Patil, G. P. and Ord, J. K. (1975), On size-biased sampling and related form-invariant weighted distributions. Sankhya, Series B, 38, 48-61.

Patil, G. P. and Rao, C. R. (1977), Weighted distributions: a survey of their applications. In Applications of Statistics. P. R. Krishnaiah (ed.), 383-405.

Patil, G. P. and Rao, C. R. (1978), Weighted distributions and size-biased sampling with application to wildlife populations and human families. Biometrics, 34, 179-189.

Rao, C. R. (1977), A natural example of a weighted binomial distribution. American Statistician, 31, 24-26.

Rao, C. R. (1985), Weighted distributions arising out of methods of ascertainment: What population does a sample represent Chapter 24 in Celebration of Statistics, The 151 Centenary Volume, A. C. Atkinson and S E Fienbwg (eds), 543-569.

Shahbaz, S., Shahbaz, M. and Butt, N. S. (2010), A class of weighted Weibull distribution. Pakistan Journal of Statistics and Operation Research, 6, 53-59.

Shakhatreh, M. K. (2012), A two-parameter of weighted exponential distributions. Statistics $\mathcal{E}$ Probability Letters, 82, 252-261.

\section{Appendix A}

Theorem 1. Let $(\Omega, \mathcal{F}, \mathbf{P})$ be a given probability space and let $H=[a, b]$ be an interval for some $\mathrm{a}<\mathrm{b}(\mathrm{a}=-\infty ; \mathrm{b}=\infty$ might as well be allowed). Let $X: \Omega \rightarrow H$ be a continuous random variable with the distribution function $F$ and let $q_{1}(x)$ and $q_{2}(x)$ be two real functions defined on $H$ such that

$$
E\left(q_{2}(X) \mid X \geq x\right)=E\left(q_{1}(X) \mid X \geq x\right) \eta(x), \quad x \in H,
$$

is defined with some real function $\eta(x)$. Assume that $q_{1}(x), q_{2}(x) \in C^{1}(H), \eta(x) \in C^{2}(x)$ and $F$ is twice continuously differentiable and strictly monotone function on the set $H$. 
Finally, assume that the equation $\eta(x) q_{1}(x)=q_{2}(x)$ has no real solution in the interior of $H$. Then $F$ is uniquely determined by the functions $q_{1}(x), q_{2}(x)$ and $\eta(x)$ particularly

$$
F(x)=\int_{a}^{x} C\left|\frac{\eta^{\prime}(u)}{\eta(u) q_{1}(u)-q_{2}(u)}\right| \exp (-s(u)) d u,
$$

where the function $s(u)$ is a solution of the differential equation $s^{\prime}(u)=\frac{\eta^{\prime}(u) q_{1}(u)}{\eta(u) q_{1}(u)-q_{2}(u)}$ and $\mathrm{C}$ is the normalization constant, such that $\int_{H} d F=1$.

We like to mention that this kind of characterization based on the ratio of truncated moments is stable in the sense of weak convergence (see, Glänzel (1990)), in particular, let us assume that there is a sequence $\left\{X_{n}\right\}$ of random variables with distribution functions $\left\{F_{n}\right\}$ such that the functions $q_{1 n}, q_{2 n}$ and $\eta_{n}(n \in \mathrm{N})$ satisfy the conditions of Theorem 1 and let $q_{1 n} \rightarrow q_{1}, q_{2 n} \rightarrow q_{2}$ for some continuously differentiable real functions $q_{1}(x)$ and $q_{2}(x)$. Let, finally, $X$ be a random variable with distribution $F(x)$. Under the condition that $q_{1 n}(x)$ and $q_{2 n}(x)$ are uniformly integrable and the family $\left\{F_{n}\right\}$ is relatively compact, the sequence $X_{n}$ converges to $X$ in distribution if and only if $\eta_{n}$ converges to $\eta$, where

$$
\eta(x)=\frac{E\left[q_{2}(X) \mid X \geq x\right]}{E\left[q_{1}(X) \mid X \geq x\right]}
$$

This stability theorem makes sure that the convergence of distribution functions is reflected by corresponding convergence of the functions $q_{1}(x), q_{2}(x)$ and $\eta(x)$ respectively. It guarantees, for instance, the 'convergence' of characterization of the Wald distribution to that of the Levy-Smirnov distribution if $\alpha \rightarrow \infty$. A further consequence of the stability property of Theorem 1 is the application of this theorem to special tasks in statistical practice such as the estimation of the parameters of discrete distributions. For such purpose, the functions $q_{1}(x), q_{2}(x)$ and, $q_{1}(x)$ specially, $\eta(x)$ should be as simple as possible. Since the function triplet is not uniquely determined it is often possible to choose $\eta(x)$ as a linear function. Therefore, it is worth analyzing some special cases which helps to find new characterizations reflecting the relationship between individual continuous univariate distributions which are appropriate in other areas of statistics.

\section{Appendix B (Linear Representation)}

An expansion for the pdf (2.2) can be derived using the series 


$$
e^{x}=\sum_{i=0}^{\infty} \frac{x^{i}}{i !}, \quad i>0
$$

Then, the density function of WEx family can be expressed as

$$
f(x ; \alpha, \xi)=\sum_{i=0}^{\infty} \frac{[1-G(x ; \xi)]^{i}}{i !} g(x ; \xi) G(x ; \xi)^{\alpha-1}[\alpha-G(x ; \xi)],
$$

or

$$
f(x ; \alpha, \xi)=\sum_{i=0}^{\infty} \sum_{j=0}^{\infty}\left(\begin{array}{l}
i \\
j
\end{array}\right) \frac{g(x ; \xi) G(x ; \xi)^{\alpha+j-1}}{i !}[\alpha-G(x ; \xi)] .
$$

Finally, we have

$$
f(x ; \alpha, \xi)=\sum_{i, j=0}^{\infty} \eta_{i, j}\left[\alpha \Lambda_{\alpha+j-1}-\Lambda_{\alpha+j}^{\prime}\right],
$$

where, $\eta_{i, j}=\frac{1}{i !}\left(\begin{array}{c}i \\ j\end{array}\right), \Lambda_{\alpha+j-1}=g(x ; \xi) G(x ; \xi)^{\alpha+j-1}$ and $\Lambda_{\alpha+j}^{\prime}=g(x ; \xi) G(x ; \xi)^{\alpha+j}$.

Also, the expression in (2.1) can be expressed as

$$
F(x ; \alpha, \xi)=\sum_{i=0}^{\infty} \sum_{j=0}^{\infty}\left(\begin{array}{l}
i \\
j
\end{array}\right) \frac{G(x ; \xi)^{\alpha+j}}{i !} .
$$

Furthermore, if $m$ is an integer, then the expression for $f(x ; \alpha, \xi) F(x ; \alpha, \xi)^{m}$ is given by

$$
f(x ; \alpha, \xi) F(x ; \alpha, \xi)^{m}=\sum_{i, j, k, l=0}^{\infty} \Upsilon_{m, i, j, k, l}\left[\alpha v_{\alpha(m+1) j+l-1}-v_{\alpha(m+1) j+l}^{\prime}\right], \quad(B-4)
$$

where,

$$
\begin{aligned}
& \Upsilon_{m, i, j, k, l}=\frac{1}{i ! k !}\left(\begin{array}{c}
i \\
j
\end{array}\right)\left(\begin{array}{c}
k \\
l
\end{array}\right) m^{i}, v_{\alpha(m+1) j+l-1}=g(x ; \xi) G(x ; \xi)^{\alpha(m+1) j+l-1} \text { and } \\
& v_{\alpha(m+1) j+l}^{\prime}=g(x ; \xi) G(x ; \xi)^{\alpha(m+1) j+l} .
\end{aligned}
$$

